\title{
Aragon transi par le chant
}

\author{
Daniel BOUGNOUX (Grenoble $)^{1}$
}

\section{Summary}

Aragon, more than any other French poet, has been interpreted in song some two hundred times. Why is music so important in his poetry? During the war (1939-1945), he himself encouraged the resistance fight with his poems, written as an inspiration to restore morale, and the memory of the nation's voice; in the communist party as well, singing was a way to come together and maintain hope. Music, generally speaking, stands beyond truth and lies, the real and the imaginary. But this oral style, cultivated by the poet-novelist, mingles with orality in a darker sense, illustrated in psychoanalysis by the sufferings of primal love, including jealousy and self-destruction, which provides the framework for the novel La mise à mort (1965).

Aragon rédigea au sortir de la deuxième guerre mondiale une série de critiques poétiques mensuelles, destinées à la revue Europe, qu'il intitula "Chroniques du Bel canto " avant de les réunir en volume sous ce titre. Mais on sait que ses poèmes, et sa popularité d'auteur, doivent une grande part de leur rayonnement aux diverses mises en chanson qui en furent faites, d'abord par Léo Ferré (Barclay 1961), puis par Jean Ferrat et une multitude d'autres interprètes, notamment Marc Ogeret, Hélène Martin, Catherine Sauvage ou Monique Morelli : la mise en musique de certains textes semblait attendre, et aller de soi au témoignage de Ferré, au point qu'on recense aujourd'hui environ deux-cents ‘ chansons > tirées des poèmes d'Aragon (Nathalie Piégay a consacré à ce phénomène d'adaptation ou de traduction un livre double, Aragon et la chanson [Piégay 2007]).

\section{Poèmes inachevés}

Il n'est pas vrai, en effet, que le texte poétique se suffise à lui-même, tant il demeure ouvert de plusieurs côtés : sur ces fameuses circonstances sans lesquelles on risque de n'entendre aux chansons que les rimes, ouvert aussi aux musiques et à la voix de ses multiples interprètes, car la poésie n'est pas faite que pour le livre imprimé, elle appelle un théâtre, un corps, une mélodie ou la diction du par cœur. Le récitant imprime physiquement le texte en lui ; de 
leur côté les compositeurs, qui ne cessent après Ferré, Ferrat ou Léonardi de mettre Aragon en musique, réussissent un tour supplémentaire d'interprétation qui semble parfois relever de la magie. Cette attraction par le chant, assez unique dans le cas d'Aragon (car les poèmes d'un Eluard, d'un Breton ou d'autres contemporains ne connurent pas la même fortune), pose plusieurs problèmes très spécifiques qu'il convient d'examiner.

Un certain inassouvissement primaire, qu'un psychanalyste qualifiera de stade oral de la libido, nourrit probablement la pulsion du chant. Il peut s'agir de réparer des contacts rompus, ou un corps morcelé, ou encore de retrouver l'inflexion, déjà recherchée par Verlaine, des " voix chères qui se sont tues ». De sorte que l'amour se chante, se met en vers, ou qu'il appelle impérieusement la vocifération mélodieuse comme une preuve orale de lui-même. "Ils étaient la romance l'un de l'autre ", dit l'épilogue d'Aurélien (Aragon 2007a, 687). Or cette oralité réveille les démons d'une incorporation ou d'une identification primaires, soit d'un amour où se mêlent intimement Eros et Thanatos. En 1965, le grand roman du chant bâti par Aragon autour de la cantatrice Fougère est aussi celui de la jalousie, et de la mise à mort. Le narrateur s'y donne par défi un infini rival et régénérateur, la voix chantante de l'autre (féminin) comme pour y mesurer et y loger - y mêler et y engloutir - son propre bel canto (Aragon 1965).

Le chant répare ou construit le sujet, il l'exalte ; la grande poésie de même doit chanter "les héros et les armes ", Arma virumque cano comme dit, citant Virgile, la préface aux Yeux d'Elsa (Aragon 2007b, 743-758) ; le chant poétique redonne confiance et croyance, les vers soutiennent l'action. "Le chant qui est la négation de la solitude poétique ", insiste Aragon dans les Chroniques du bel canto (Aragon 1979, 156). L'hymne ne propose pas une connaissance à distance, il a valeur pratique et non pas historique ni théorique, et il s'élabore au contact des hommes. Son rythme, et l'accouplement de ses rimes, exaltent la croyance et préfigurent concrètement l'utopie d'une harmonie sociale annoncée, à la façon dont le chant signifiait à l'étage individuel et pour le couple amoureux une promesse d'harmonie. C'est ainsi que la Révolution autant que l'amour se chantent, d'un chant facteur de croyance, donc de croissance; consubstantielle au groupe, au nouage du nous, la poésie est la vitamine du corps collectif.

"Toute musique me saisit..." (Aragon 1956,154) : lui-même semble transi par ses poèmes, chanteur par ses voix traversé. Musicien déconcertant, Aragon suspend parfois ses paroles si précises, et si capables de remuer nos propres élans, au bord d'un silence qui donne également à penser. Dans Le fou d'Elsa publié en 1963, et qui retrace l'agonie du royaume de Grenade en 1492, le Medjnoûn est tombé aux mains des bourreaux ; les policiers aussi ont besoin des poètes, et ils pressent celui-ci de chanter :

Le chant ne s'accommode pas qu'on mente

Le chant disait-il n'est commandement

Eux disaient Chante on te dit chante

Ils l'ont tant frappé qu'il chanta [...]

Mais eux n'entendaient que les rimes 
Ils disaient Chante chante encore

C'était le mois de mouharram

Et par la porte on pouvait voir

Sur le monde noir de son drame

Pleuvoir Pleuvoir Pleuvoir Pleuvoir

(Aragon 1963, 319-320)

On vérifie sur de tels passages à quel point l'érotique, la poétique et la politique tournent chez Aragon dans un cercle. Le chant berce et entraine, par-delà tout réalisme ; il retend le corps individuel, mais aussi social : « Pour un chant (national) » donne son titre à un poème des Yeux d'Elsa adressé en août 1941 au poète (un peu trop éthéré) Alain Borne, par lequel Aragon résume alors son art poétique et son programme. Ce chant n'est jamais plus fort qu'à l'époque de la France en morceaux, quand il permet à l'individu autant qu'au collectif d'incorporer l'objet perdu.

\section{Promesse de communauté}

Force de mémoire par sa métrique et sa sémantique, force vitale de cohésion (nationale, révolutionnaire autant qu'amoureuse car le couple mais aussi le pays, la patrie, le parti, les lendemains ou l'action partagée, également se chantent), ce chant marque l'Histoire et le territoire, où il est promesse de communauté. Ou mieux que promesse, évidence du lien et actualisation de l'être-ensemble.

Au niveau amoureux par exemple, les premiers chants du Crève-cœur disaient les amants séparés, et cette plainte lyrique semblerait conventionnelle si à travers celle-ci ne sourdaient d'autres déchirures... Que l'amour par lui-même exige la romance, cela est dit dans Aurélien où l'amour pour Bérénice naît d'abord de l'évidence de ce qui chante en elle, « une présence. Une absence. Les deux à la fois. Une chanson » (Aragon 1945, 145), avant de se conclure par les phrases déchirantes de l'épilogue : "Vous êtes tout ce qui a jamais chanté dans ma vie." (Aragon 1945, 688) Le chant amoureux appelle l'unisson, et l'harmonie réalisée des sons prépare celle des corps. Ou plus précisément : la mélodie répare l'unité du corps morcelé, au niveau individuel comme à celui du couple, ou de la nation.

Pour remembrer la France de 1940, le chant opère par le rappel des mètres et par la multiplication des allusions historiques ; fouillant un passé profond, il prépare l'avenir : on se groupe, on s'identifie, on s'active autour du chant. Le patriote et le militant, comme l'amoureux, s'arrachent au chétif petit moi pour s'agréger, par la force coagulante du chant, au corps collectif en gestation. Le chant ne se réfute pas, il n'argumente pas - non plus que l'amour ou le rêve : il mobilise, enveloppe et saisit. Le chant n'a pas simplement pour objet ‘ les héros et les armes , il donne des armes aux hommes qui n’en ont pas. Très délibérément, au-delà du double-entendre et de la savante contrebande pratiqués durant les années de guerre (qui exigeaient la sagacité du récepteur), Aragon aura visé par sa poésie ce ravissement 
primaire. Car l'évidence du chant - chanter dans les supplices, chanter pour (se) redonner du courage, ou pour rejoindre ses camarades - est par elle-même message, et source d'énergie. Que disait «Les lilas et les roses » du Crève-coeur par exemple (poème publié dès juillet 1940 dans Le Figaro), sinon que sous la botte allemande la France était capable de donner de la voix ? Le premier acte de résistance consistait à sauver un langage ou un chant national contre la crétinisation escomptée par Vichy.

Plus tard, on croisera dans Le Fou d'Elsa (1963) la déploration d'un musicien aveugle, comme un de ces nombreux miroirs de la condition de l'auteur disposés en passant. " Toute musique me saisit " (Aragon 2007c, 206). Ce saisissement, et le motif de la cécité amoureuse ou politique ne feront que grandir dans les textes de cette dernière période, autocritique, qui va du Fou à Théâtre/Roman (Aragon 1974). Aragon y interroge un réalisme (musical ?) devenu fort problématique, à partir du moment où le militant préfèrera toujours le torrent du lyrisme ou de la fabulation qui fait vivre dans l'exaltation, mais qui distille un charme peu propice à l'esprit critique.

Longtemps, les révolutionnaires chantèrent la colère et la guerre nécessaire, en reléguant l'amour, chose non-bolcheviste, au rang d'un luxe bourgeois. Aragon, qui signe sous l'occupation François La Colère, corrige ou complète cette passion en s'enivrant de lyrisme amoureux, et il voit dans la chance du chant, comme dans le mythe, un pôle de transcendance et d'organisation.

\section{Les démons de l'oralité}

L'amour et le réalisme pourtant tirent en sens contraire s'il est vrai que l'amour, au moins pour Aragon, implique une illusion partagée et une servitude volontaire, toujours en quête de complétude et d'identification positive pour réparer notre inachèvement (catégorie majeure sur les trois plans politique, érotique, poétique). Si dans La mise à mort, ce ‘ roman du réalisme , qui fait une large place au bel canto et à la jalousie, Alfred prenant le nom d'Anthoine est devenu réaliste par amour, sa passion le conduira à s'effondrer dans la folie, dernier mot d'un ouvrage qui interdit de voir dans le couple ici mis en scène une pédagogie du bonheur social, ou la première cellule de la société à venir.

Qui parle ou chante dans La mise à mort ? Il devient vite évident, dès la présentation dithyrambique qu'Aragon fait de la cantatrice à laquelle il compare Colette au passage "C'est ça la voix de Fougère. [...] j'y crois. Cet art de s'effacer plus grand que tout autre, l'art des choses banales qui vous entrent dans le cour sans qu' on semble y avoir rien ajouté " (Aragon1965, 16) - que l'auteur ne prête à l'autre son propre chant que pour mieux s'entendre, et s'émerveiller lui-même. La distribution des rôles dans La mise à mort sert à externaliser la source de ce chant, par une autoréférence indirecte ou coudée ; en Narcisse rusé ou consommé, Aragon non seulement veut se voir mais s'entendre en Fougère.

L'oralité pourtant endosse un autre sens, plus noir mais également pertinent concernant notre auteur ; on parle en psychanalyse, disais-je, d'amour oral pour désigner un premier 
stade de la libido, lié à la bouche et qui détruit son objet. Cette ravageuse oralité se marque notamment dans les conduites d'identification (le sujet veut s'incorporer l'objet de son amour), de jalousie morbide (l'oralité ne laisse pas son objet calmement exister à distance), de narcissisme (la dépendance amoureuse est vécue selon une alternative de vie ou de mort, un moi rageur s'approprie l'autre, en mettant à mort ses rivaux). Ces démons semblent bien attestés dans l'intrigue du roman tramé autour de la voix de Fougère et intitulé (titre révélateur) La mise à mort (1965), soit, résume Aragon ici ou là, un roman sur la jalousie : Alfred y est jaloux d'Anthoine, Anthoine jaloux de Fougère, ou plus précisément des pouvoirs de son chant, de son opéra : "Elle chante, et j'ai cessé d'être pour ne faire que suivre. " (Aragon $1965,38)$ < Je suis > se conjugue pour Anthoine à la première personne du verbe suivre.

Ce roman ne nous cache rien des ravages d'un amour oral, qu'un psychanalyste dirait prégénital ; cet amour dévorant, tumultueux, inutilement paroxystique conduit à la tragédie ou, en effet, au crime. Opérateur de liaison, la pulsion de chant comble l'inassouvissement oral primaire ; c'est le chant qui répare les contacts rompus et le corps morcelé, qui éloigne les fantasmes d'abandon, de solitude et de perte. Cette oralité partout active sous sa plume réveille pourtant les démons d'une incorporation dangereuse pour l'autre ; de même que la pulsion orale détruit l'objet aimé en le dévorant, l'identification et le désir fusionnel ne laissent pas les individus intacts ; l'amour dans ses manifestations orales mêle intimement Eros avec Thanatos. Le grand roman de Fougère bâti par Aragon pour exalter par son chant le sien propre est aussi celui de la jalousie, de la dépossession et de la psychose. Quel paradoxe d'avoir lié, dès le début de ce roman, sa conversion réaliste aux pouvoirs de ce chant, et de son amour ! Le dernier mot de notre ouvrage, ' folie , (en écho au précédent Fou d'Elsa), interdit de tirer de cet amour toujours en proie à l'incomplétude une pédagogie du réalisme.

Le chant d'ailleurs se moque bien du principe de réalité. Preuve de l'union par l'unisson, la communication lyrique retisse un lien ou une attache primaire très en deçà, ou au-delà, du vrai et du faux, du bien et du mal. Le réalisme n’est pas son fort. Le chant exalte le militant ou l'amoureux qui s'engagent moins au terme d'un raisonnement que pour le plaisir de chanter ensemble; en amour comme en politique, l'hymne est irréfutable, il échappe à la discussion rationnelle; et c'est tout le service qu'un grand parti attend du poète : que le torrent de son lyrisme ou de sa fabulation suscite l'enthousiasme, et ravive un charme.

Porté par la musique, Aragon ranime au fil de ses poèmes l'obscur chamanisme de la possession et de la transe. Les trafics de l'oralité mis en scène dans La mise à mort brouillent l'origine d'une voix qui, ainsi placée en transcendance, peut s'avérer persécutrice ; c'est sous son emprise qu'Alfred s'est métamorphosé en Anthoine : «Est-ce que je lui déplaisais tant, me trouvait-elle si laid que j'eusse à renoncer à mon apparence ? Qu'est-ce qu'elle avait donc chanté, ce jour-là, du ciel ou de l'enfer ? [...] Quand Fougère chante, je tombe toujours. Elle me déchire. " (Aragon 1965, 19) Cet aspect du chant-qui-rend-fou est développé par l'histoire subséquente du roi danois Erik Ejegod (Aragon 1965, 268-269), que la musique poussa au crime, et les mentions incidentes de Johnny Hallyday ou des Beatles (Aragon 1965, 276). Après quoi «Le Carnaval ", traversé de musiques aux fantastiques ressources de contrebande - «Quand je pense, Mozart, Beethoven... quels merveilleux contrebandiers!» 
(Aragon 1965, 276) -, médite aussi sur la tromperie d'un art qui fit naître chez Pierre des promesses que la jeune pianiste Bettina ne pouvait tenir.

\section{"Un grand mouchoir lyrique »}

Un réalisme musical n'est-il pas une contradiction dans les termes ? La musique qui nous berce, en fermant toute discussion, peut aussi nous berner. Les jeux de la rime et des rythmes, pas plus que les charmes de la fabulation, ne servent efficacement l'esprit critique. Ils exaltent, et ravissent, mais engendrent l'hypnose plutôt que la claire évidence d'une preuve.

La blessure ontologique de l'amour, la jalousie posée en principe, la constitution ' courtoise , d'Elsa en objet perdu, une certaine folie bien réelle disloquent la prose des derniers romans comme celle de poèmes qui n'ont plus rien de mélodieux, mais cherchent à rejoindre l'origine du souffle, une cacophonie panique, un essai de la voix. L'intérêt avec Aragon est que ce passage aux limites, aux échardes, aux esquisses, loin de détruire la musique la fasse renaître sous une forme neuve. Dans les poèmes dadaïstes de même, la volonté de "mettre le pied sur la gorge de sa propre chanson " (Aragon 1953, 128) (selon l'illustre programme de Maïakovski) n'abolissait pas le sortilège poétique, toujours perceptible dans les réductions aussi drastiques que le poème "Suicide » qui semble se contenter de signer l'alphabet - tout en lui donnant une certaine respiration.

N'écoute que ma voix les chansons

mentent le désespoir cache sous son grand

mouchoir lyrique

la véritable horreur du monde [...] (Aragon 2007b, 489)

écrit Aragon à Elsa au début de leur relation, dans un poème de 1929 demeuré longtemps inédit.

Chacun écoute les poètes comme ça lui chante. On préfère aimer dans le chant d'Aragon ce qui berce, et Jean Ferrat contribua plus que Ferré peut-être à sa popularité. Mais le "grand mouchoir» n'étouffe jamais tout à fait ce qui perce, le monde réel dans son horreur. Musicien grinçant ou déconcertant, chanteur par ses voix traversé, Aragon tire son pouvoir d'enchantement de deux chaos grondants, les guerres du monde réel et d'une Histoire toujours en gésine, et les discordes intimes d'un monde intérieur et d'une identité en proie à de multiples démons, jusque dans l'amour - qui ne met pas forcément fin aux tourments. Mais quels qu'aient été les déchirements individuels et collectifs éprouvés du début à la fin de son orageuse existence par ce prince du chant - qui avait l'oreille absolue -, sa force fut d'orchestrer savamment ses ruptures, de chanter jusqu'au bout ses échecs et d'en tirer un somptueux opéra. 


\section{Note}

1 Daniel Bougnoux, philosophe, éditeur des Euvres romanesques complètes d'Aragon dans la bibliothèque de la Pléiade (cinq volumes), est professeur émérite à l’Université Grenoble Alpes.

\section{Bibliographie}

Aragon, Louis : Aurélien. Paris : Gallimard, 1945.

Aragon, Louis : L'Homme communiste. Vol. 2. Paris : Gallimard, 1953.

Aragon, Louis : Le roman inachevé. Paris : Gallimard, 1956.

Aragon, Louis : Le fou d'Elsa. Paris : Gallimard, 1963.

Aragon, Louis : La mise à mort. Paris : Gallimard, 1965.

Aragon, Louis : Théâtre/Roman. Paris : Gallimard, 1974.

Aragon, Louis : Chroniques du bel canto (1946). Textes repris dans Chroniques de la pluie et du beau temps. Paris : Les Éditeurs français réunis, 1979.

Aragon, Louis : Aurélien. Paris : Gallimard, 2007. [=Aragon 2007a]

Aragon, Louis : Euvres poétiques complètes. Vol. 1. Paris : Gallimard, 2007. [=Aragon 2007b]

Aragon, Louis : Euvres poétiques complètes. Vol. 2. Paris: Gallimard, 2007. [=Aragon 2007c]

Hirschi, Stéphane : "Aragon et la chanson ». In : Bougnoux, Daniel (éd.) : Aragon, la parole ou

l'énigme. Paris : Éditions de la Bibliothèque publique d'information, 2005. En ligne, généré le

07.12.2020 : http://books.openedition.org/bibpompidou/823 (consultation 10.12.2020).

Piégay, Nathalie : Aragon et la chanson. 2 vol. Paris : Textuel, 2007.

Zumthor, Paul : Introduction à la poésie orale. Paris : Éditions du Seuil, 1983.

\section{Discographie}

Ferrat, Jean : Ferrat chante Aragon. Barclay 80443, 1971 (33 tours).

Ferrat, Jean : Ferrat chante Aragon. Temey 2400502, 1994 (CD).

Ferré, Léo : Léo Ferré chante Aragon. Barclay 841-271-2, 1961 (33 tours).

Ogeret, Marc : Marc Ogeret chante Aragon. Vogue 9455, 1967 (33 tours). 